\title{
Towards Energy-Efficient Ventilation in Buildings: Development of the Smart Window Ventilation System
}

\author{
Behrang Chenari, João Dias Carrilho, Gustavo Botte, and Manuel Gameiro da Silva
}

\begin{abstract}
As the building sector is responsible for a large portion of total primary energy consumption in developed countries, it is crucial to improve energy efficiency of buildings' systems. Heating, ventilation, and air conditioning systems play a noticeable role in energy consumption of buildings, therefore, developing efficient strategies for ventilation not only can provide comfortable indoor climate but can also mitigate energy consumption in buildings, which, consequently, will lessen the associated environmental impacts. This paper presents development steps of an intelligent window-based hybrid ventilation system in an ongoing research and development project at the University of Coimbra, known as the Smart Window project. Firstly, the motivation and objectives of the project are provided. Secondly, the current state of development of the project as well as the test condition and location in an Indoor Live Lab is presented. Finally, the future steps of development of the project accompanied by the expecting results are presented and discussed.
\end{abstract}

Index Terms-Control strategies, demand-controlled ventilation, energy efficiency, hybrid ventilation, indoor environmental quality, Smart Window.

\section{INTRODUCTION}

Nowadays, energy demand is continuously increasing worldwide, therefore, the negative environmental impact resulting from energy production and consumption has become a major public concern. The building sector is one of the biggest contributors to energy consumption in the world, which is responsible for a large portion of total primary energy consumption in developed countries. Heating, ventilation, and air conditioning systems play a noticeable role in energy consumption of buildings, thus, the design and development of new energy-efficient strategies and products for ventilation systems in buildings is crucially essential. Currently, there is ongoing research promoting energy saving potential in buildings in the framework of the EU Energy Performance of Building Directive (EPBD) [1], which has focused on energy efficiency in buildings. Some authors investigated new energy-efficient solutions for improving ventilation systems in buildings. On the other hand, in the last decade, many studies have concentrated on improving indoor

Manuscript received March 20, 2015; revised September 16, 2015. This work was partially supported by the project Smart Window CENTRO-07-0202-FEDER-034123.

Behrang Chenari, João Dias Carrilho, and Manuel Gameiro da Silva are with the ADAI, LAETA, Department of Mechanical Engineering, University of Coimbra, Polo II, 3030-788, Coimbra, Portugal (e-mail: behrang.chenari@student.dem.uc.pt, joao.carrilho@dem.uc.pt, manuel.gameiro@dem.uc.pt).

Gustavo Botte is with the WSBP Electronics, Lda., Rua Pedro Nunes IPN TecBIS, 3030-199, Coimbra, Portugal (e-mail: gsb@wsbp.eu). air quality (IAQ) in buildings. There is a tight correlation between ventilation and IAQ, and the reduction of energy consumption associated to ventilation cannot be an excuse to neglect the provision of a suitable indoor climate. Therefore, in developing energy-efficient solutions for ventilation systems, maintaining the indoor climate at an acceptable level must be considered.

As aforementioned, ongoing research is focused on developing energy-efficient ventilation models for buildings. Some studies have only focused on natural ventilation as a passive method in order to make the process of ventilation more efficient [2]-[5]. Some authors used natural ventilation elements such as atria [6], wind towers [7], double-skin facades [8] as well as ventilation openings and windows [9] to provide required ventilation by natural driving forces, namely wind and buoyancy forces. Additionally, some studies investigated energy savings from utilizing different principles of natural ventilation, such as single-sided [3], cross ventilation [10] and stack effects [11]. But, as natural ventilation is not always available, say, sufficient to provide required ventilation rate, more recent studies [12], [13] focused on hybrid ventilation [14]. Hybrid ventilation will exclude the drawbacks of both systems, whereas including their advantages [15].

Moreover, several authors have developed ventilation control strategies that provide acceptable indoor climate quality levels and lowers energy consumption. They believe that the presence of control systems can increase the influence of hybrid and natural ventilation in improving energy saving in buildings, while maintaining a suitable indoor climate. These authors conducted research activities in developing new control strategies to employ natural cooling and ventilation. For instance, Homod et al. [16] developed control strategies, which can predict when mechanical ventilation is required in order to maintain acceptable IAQ. In addition to predictive control strategies, rule-based and demand-controlled ventilation (DCV) strategies, as used in [17]-[21], have significant effects on improving energy efficiency of ventilation systems.

\section{Motivation and Objectives}

As people spend most of their time inside buildings, it is very important to maintain an adequate IAQ, in addition to thermal comfort, especially for office buildings and schools in which people are working, learning and studying, and where the level of personal control over their indoor environment can be very limited. IAQ and building ventilation are intimately related because ventilating indoor spaces with outdoor air is perhaps the most often used mechanism to dilute pollutants generated inside the building. Ventilation is 
the key issue for providing suitable IAQ, as it is the process of replacing stale indoor air by fresh outdoor air. It can be performed either by mechanical means (mechanical ventilation) or natural driving forces (natural ventilation) or even a combination of them (hybrid ventilation). Employing natural ventilation in buildings results in the reduction of energy consumption, while maintaining an acceptable level of IAQ. Therefore, developing ventilation control strategies that can predict when natural ventilation is available and sufficient, and select between natural and mechanical ventilation as needed, are desirable.

Although recent studies have shown research activities in development of new control strategies to employ natural cooling and ventilation, there is still an obvious gap in the literature about employing intelligent window-based ventilation control strategies, not only for increasing the share of natural ventilation but also for integrating mechanical ventilation to compensate drawbacks of natural ventilation. This ongoing research and development project aims to fulfill this gap in literature.

This paper presents development steps of an ongoing research and development project, aiming at developing a novel product, the Smart Window, and test a number of ventilation control strategies in order to find the most energy-efficient control strategies that provide acceptable indoor climate.

\section{SMART Window: CURRENT State OF DEVElopMENT}

\section{A. Project Description}

In this project, a Smart Window system that allows the use of hybrid ventilation, is being developed to employ the advantages and exclude the drawbacks from both natural and mechanical ventilation. Moreover, several ventilation control strategies are being developed and tested using Smart Window in order to find out the best strategies that can provide an appropriate indoor climate with lower energy consumption. The control strategies, which are based on indoor and outdoor environmental parameters, such as concentration of $\mathrm{CO}_{2}$, indoor and outdoor temperature difference, wind speed and its orientation, are defined in a way to use natural ventilation as long as possible, otherwise, putting the mechanical system on circuit to provide the required space ventilation. The Smart Window is accompanied by a mechanical ventilator as well as a control system.

\section{B. Prototypes of the Mechanical Boxes}

By now, two prototypes of mechanical boxes are built. Fig. 1 shows the first prototype, which is driven by a tangential blower and is currently being installer in the Indoor Live Lab (I2L) [22] facility, located in Department of Mechanical Engineering at University of Coimbra.

Fig. 2 shows the second prototype, which is based upon a centrifugal blower and automatically operable window. The automatically operable windows will have a positive impact in IAQ and energy consumption, while permitting the manual override by the office occupants.

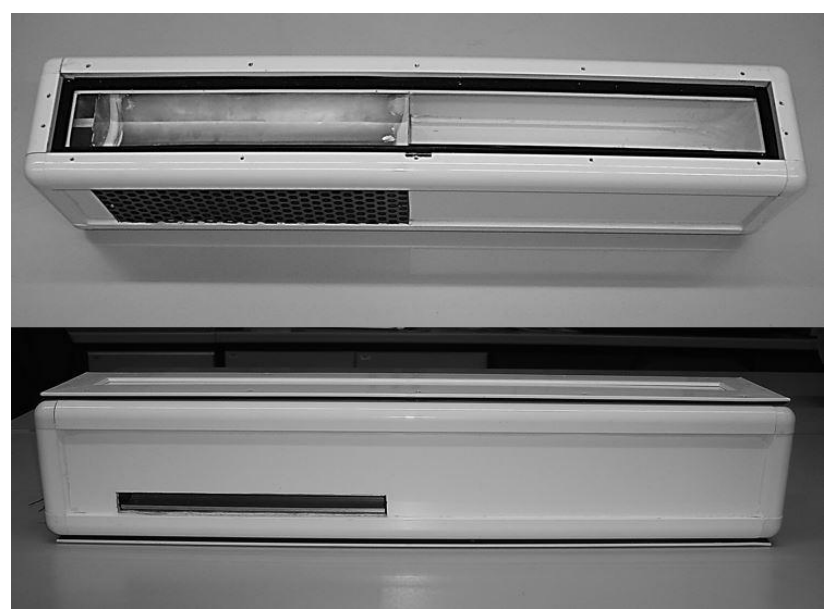

Fig. 1. Prototype of the mechanical ventilation subsystem of the Smart Window hybrid ventilation system (tangential blower).
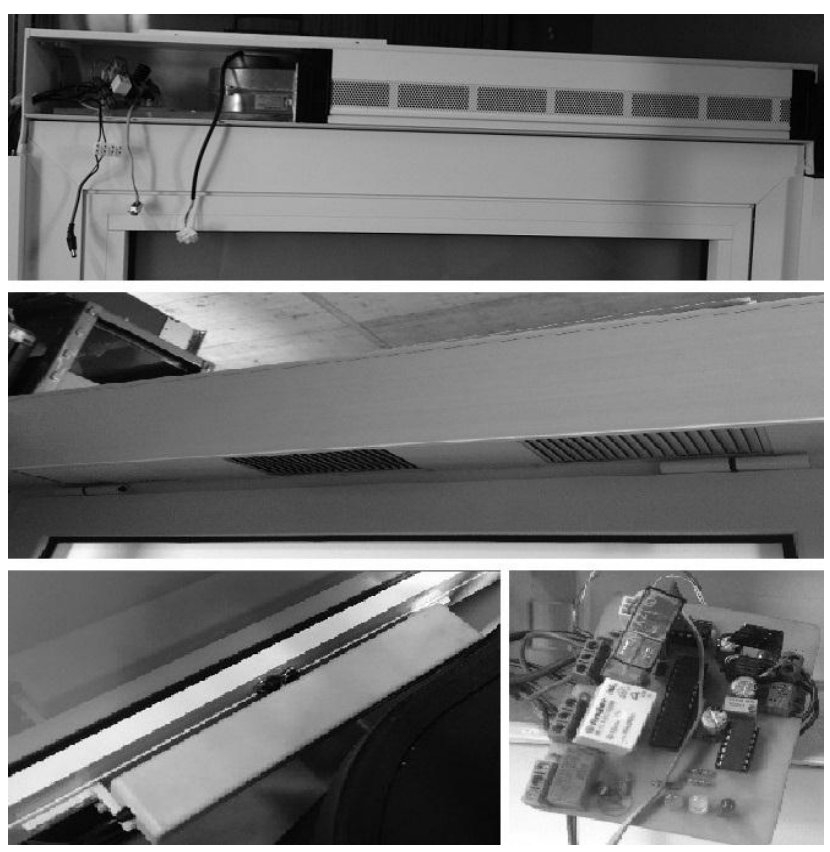

Fig. 2. Prototype of the mechanical ventilation subsystem of the Smart Window hybrid ventilation system: centrifugal blower (top); automatically operable window actuator (lower left) and control board (lower right).

\section{Control Strategies}

A number of control strategies were identified as potentially suitable, based on indoor environment parameters and outdoor weather as input data. In particular, the indoor concentration of $\mathrm{CO}_{2}$ is used to see whether ventilation is required or not. Temperature difference between indoor and outdoor $(\Delta \mathrm{T})$, wind speed (WS) and its direction (D) are used to detect the availability of natural ventilation.

In the control strategies, the availability of buoyancy-driven natural ventilation is evaluated according to (1), whereas (2) evaluates the availability of wind-driven natural ventilation.

$$
Q=C A \sqrt{2 g H \frac{T i-T o}{T i}}
$$

where $Q$ represents the ventilation airflow rate $\left(\mathrm{m}^{3} / \mathrm{s}\right), C$ is the discharge coefficient for opening (typically 0.62), $A$ indicates the cross section area of opening $\left(\mathrm{m}^{2}\right), g$ is the gravitational 
acceleration $\left(\mathrm{m} / \mathrm{s}^{2}\right), H$ represents the height from midpoint of lower opening to midpoint of upper opening $(\mathrm{m}), \mathrm{Ti}$ is the average indoor temperature $(\mathrm{K})$ and $T o$ is the outdoor temperature in $(\mathrm{K})$.

$$
Q=K A V
$$

where $Q$ represents the ventilation airflow rate $\left(\mathrm{m}^{3} / \mathrm{h}\right), A$ indicates the cross section area of opening $\left(\mathrm{m}^{2}\right)$ and $K$ is coefficient of effectiveness. This coefficient varies with the angle between wind direction and the facade opening. For instance, if the angle at which wind hits the building is $45^{\circ}$, then the coefficient is estimated to be around 0.4 , whereas, if wind hits the building perpendicularly, the coefficient is becomes about 0.8 .

According to all the aforementioned parameters, various rule-based (if CONDITION, then ACTION) control strategies were reported in the literature (e.g., see Ref. [23]), in which indoor and outdoor environmental parameters are the conditions and different operations of window and fan are the actions.

The control strategies start with simple $\mathrm{CO}_{2}$-based demand-controlled ventilation (DCV) strategies in the beginning and, going forward, reach more advanced control strategies, considering all the parameters and more advanced operations of window and fan. Table I presents characteristics of some of the developed controls from simple to advanced strategies. As shown, different input parameters and different operations of window and fan, define the control strategy either simple or advanced. Moreover, Fig. 3 demonstrates a simple control strategy in a flowchart.

TABLE I: THE CHARACTERISTICS OF CONTROL STRATEGIES

\begin{tabular}{cccc}
\hline \hline $\begin{array}{c}\text { Rule-base } \\
\text { d control }\end{array}$ & $\begin{array}{c}\text { Mechanical } \\
\text { ventilation operation }\end{array}$ & $\begin{array}{c}\text { Natural ventilation } \\
\text { operation }\end{array}$ & $\begin{array}{c}\text { Input } \\
\text { parameters }\end{array}$ \\
\hline Simple & On/Off & Open/Close $(\mathrm{O} / \mathrm{C})$ & $\mathrm{CO}_{2}$ \\
$\cdot$ & Leveling speed & Leveling O/C & $\mathrm{CO}_{2}$ \\
. & Modulating speed & Modulating O/C & $\mathrm{CO}_{2}$ \\
$\cdot$ & On/Off & O/C & $\mathrm{CO} 2+\Delta \mathrm{T}+\mathrm{D}$ \\
$\cdot$ & Leveling speed & Leveling O/C & $\mathrm{CO} 2+\Delta \mathrm{T}+\mathrm{D}$ \\
Advanced & Modulating speed & Modulating O/C & $\mathrm{CO} 2+\Delta \mathrm{T}+\mathrm{D}$ \\
\hline \hline
\end{tabular}

\section{Installation and Test}

As aforementioned, the first prototype is being installed and tested in the I2L. The I2L is equipped with various types of instruments and sensors to measure and monitor energy consumption and IEQ elements, such as thermal comfort, IAQ, lighting, sound level and so on. Fig. 4 shows a set of instruments and sensors installed in the I2L. They are all connected to a computer, which continuously monitors all these elements. Moreover, Fig. 5 depicts a daily monitored IEQ elements in the I2L. The graphs show the changes in each element during a full day, whereas the numbers shows the current value of each element.

For thermal comfort, the Predicted Mean Vote (PMV) thermal comfort index is used. This index was originally introduced by Fanger [24] and has been adopted in current international standards such as, for instance, ISO 7730 [25]. It predicts how a large number of people would vote, on average, about their perception of thermal comfort in a given indoor environment. The scale goes from -3, corresponding to the sensation of cold, with 0 corresponding to a neutral sensation, and up to +3 , corresponding to the sensation of hot. Similarly, for IAQ, the concentration of $\mathrm{CO}_{2}$ is being measured as an indicator of the balance between the ventilation rate and the generation rate of occupant-related pollutants. This shows when outdoor flow rate is required to ventilate the space and provide acceptable IAQ level. For instance, as recommended by many IAQ standards and building regulations, $1000 \mathrm{ppm}$ is normally being considered as a threshold for indoor $\mathrm{CO}_{2}$ concentration, from which ventilation is required in order to decrease the concentration of pollutants.

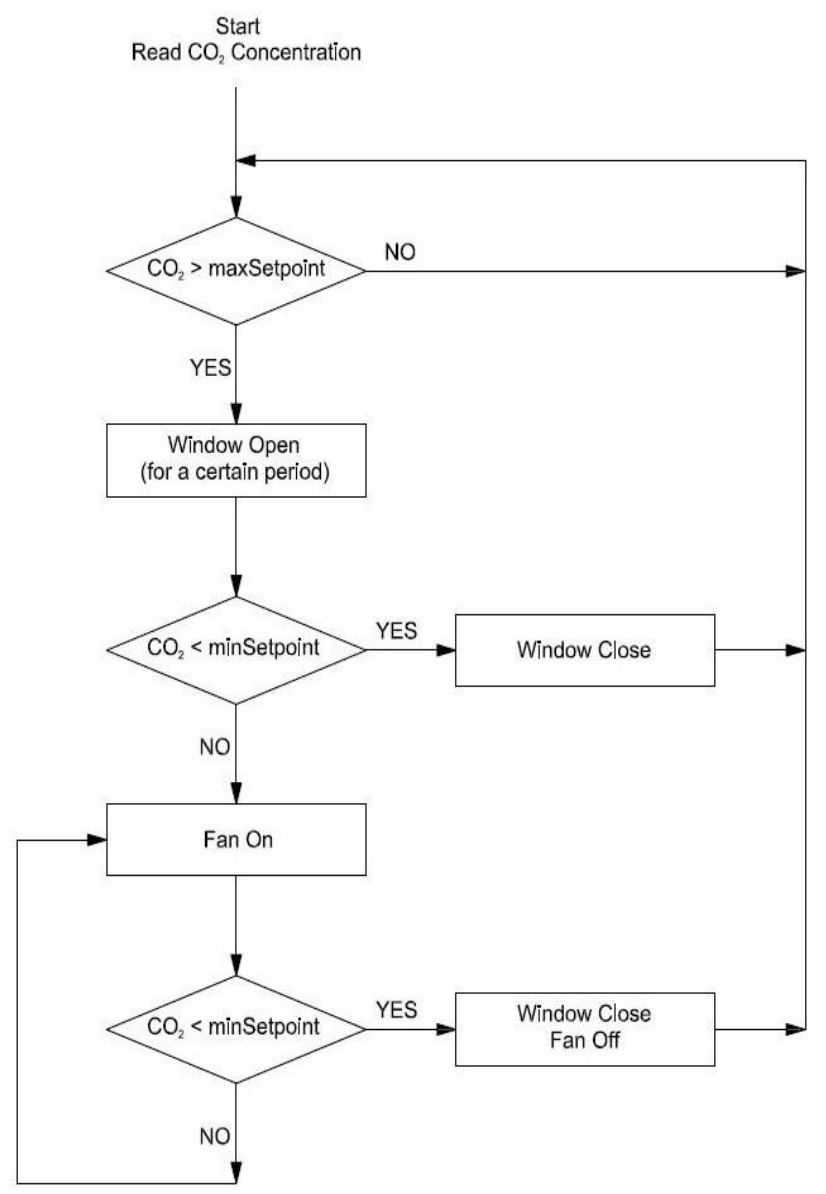

Fig. 3. Simple $\mathrm{CO}_{2}$-based control strategy.

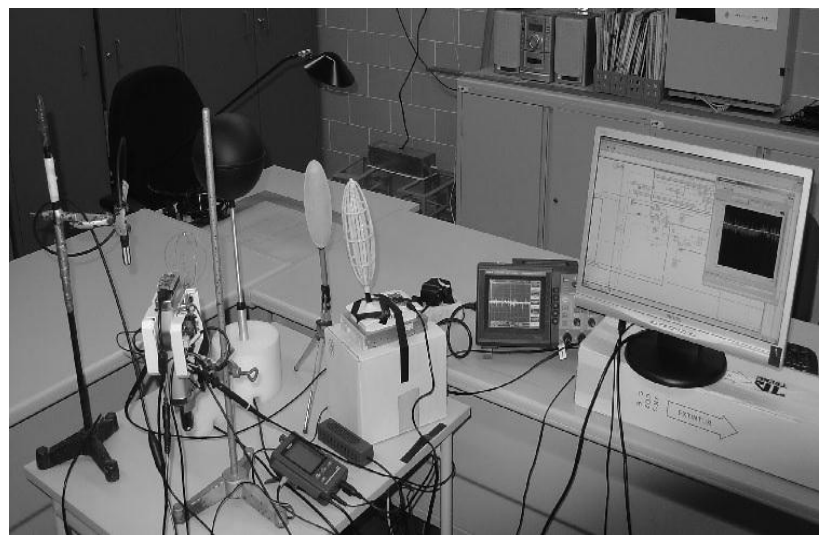

Fig. 4. Set of instruments and sensors at the I2L.

Installing the prototype in the $\mathrm{I} 2 \mathrm{~L}$ allows us to test all the control strategies, using the available instruments and sensors. 
Besides the IAQ and thermal comfort assessment, it is possible to assess the energy consumption associated to each control strategy.

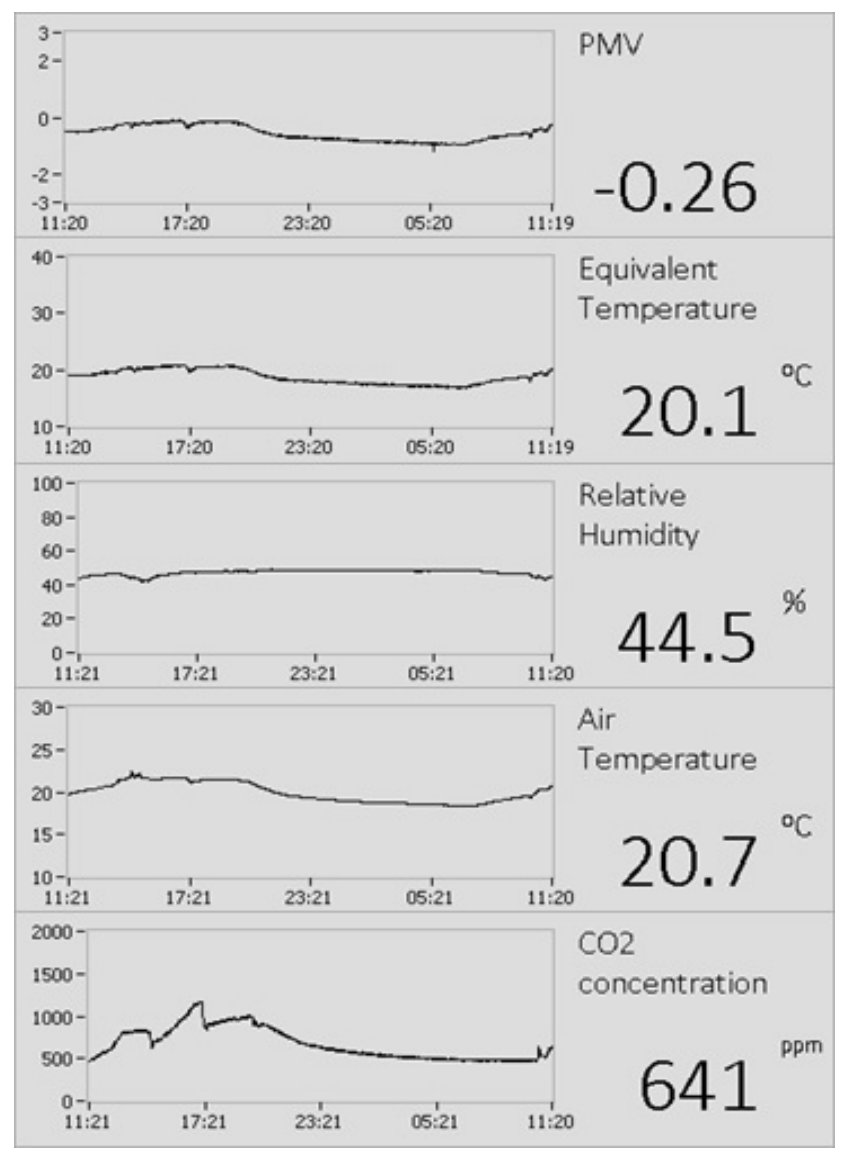

Fig. 5. Monitored IEQ indicators in the I2L.

\section{SMART Window: FutURE DEVELOPMENTS}

Besides the completed tasks, there are some more tasks in the project work plan. This section presents the future developments of the Smart Window project.

\section{A. Prototypes of Mechanical Boxes}

The new prototypes of mechanical boxes are under development, using other types of fan (axial, tangential and centrifugal) with different airflows. This is to assess airflow rate and fresh air penetration inside the space as well as the associated noise and energy consumption by each model. With regard to these data, it is possible to optimize all these parameters.

\section{B. Occupancy Counter}

An occupant counter is being developed to be installed at the entrance door of the $\mathrm{I} 2 \mathrm{~L}$. This is capable of detecting people entering and leaving the room with two infrared beams, therefore, it can calculate how many people are in the room at any moment in time. Fig. 6 shows the infrared occupancy counter set being developed. This enables the adjustment of the ventilation rate based on real occupancy level, which will help preventing energy wastage. Moreover, a comparative energy performance analysis of different ventilation model (constant volume of airflow, $\mathrm{CO}_{2}$-based DCV and occupancy-based DCV) is being carried out.

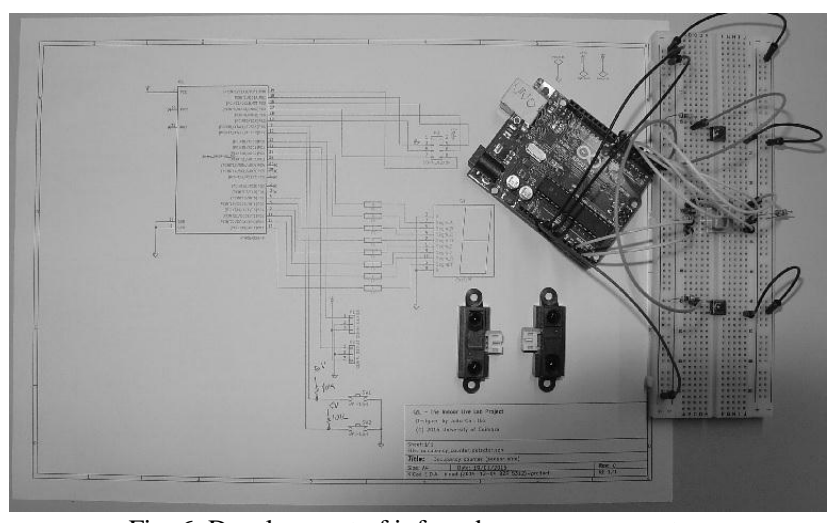

Fig. 6. Development of infrared occupancy counter.

\section{CONCLUSIONS}

Ventilation is the key issue for providing suitable IAQ, as it is the process of replacing stale indoor air by fresh outdoor air. It is also responsible for a notable percentage of energy consumption in both residential and commercial buildings. Therefore, improving ventilation systems is vital both for improving energy efficiency in buildings and for providing acceptable IAQ. The Smart Window project described in this paper integrates hybrid ventilation to the building facade in order to employ the advantages and exclude the drawbacks from both natural and mechanical ventilation. The paper presented the current development steps of the intelligent window-based hybrid ventilation system in an ongoing research and development project, at University of Coimbra. The motivation and objectives behind this project as well as the current development, ongoing tasks and future work plans were presented and discussed.

\section{REFERENCES}

[1] Directive 2010/31/EU of the European Parliament and of the Council of 19 May 2010 on the energy performance of buildings, Official Journal of the European Union, vol. 153, issue 13, June18, 2010.

[2] C. Allocca, Q. Chen, and L. R. Glicksman, "Design analysis of single-sided natural ventilation," Energy Build, vol. 35, no. 8, pp. 785-795, Sep. 2003.

[3] T. S. Larsen and P. Heiselberg, "Single-sided natural ventilation driven by wind pressure and temperature difference," Energy Build, vol. 40, no. 6, pp. 1031-1040, Jan. 2008.

[4] R. Yao, B. Li, K. Steemers, and A. Short, "Assessing the natural ventilation cooling potential of office buildings in different climate zones in China," Renew. Energy, vol. 34, no. 12, pp. 2697-2705, Dec. 2009.

[5] I. Oropeza-Perez and P. A. Østergaard, "Potential of natural ventilation in temperate countries - A case study of Denmark," Appl. Energy, vol. 114, pp. 520-530, 2014.

[6] S. D. Ray, N.-W. Gong, L. R. Glicksman, and J. A. Paradiso, "Experimental characterization of full-scale naturally ventilated atrium and validation of CFD simulations," Energy Build, vol. 69, pp. 285-291, Feb. 2014

[7] C. Tantasavasdi, J. Srebric, and Q. Chen, "Natural ventilation design for houses in Thailand," Energy Build., vol. 33, no. 8, pp. 815-824, Oct. 2001.

[8] E. Gratia and A. de Herde, "Natural ventilation in a double-skin facade," Energy Build, vol. 36, no. 2, pp. 137-146, Feb. 2004.

[9] M. Z. I. Bangalee, S. Y. Lin, and J. J. Miau, "Wind driven natural ventilation through multiple windows of a building: A computational approach," Energy Build, vol. 45, pp. 317-325, Feb. 2012.

[10] A. D. Stavridou and P. E. Prinos, "Natural ventilation of buildings due to buoyancy assisted by wind: Investigating cross ventilation with computational and laboratory simulation," Build. Environ., vol. 66, pp. 104-119, 2013. 
[11] X. Q. Zhai, Z. P. Song, and R. Z. Wang, "A review for the applications of solar chimneys in buildings," Renew. Sustain. Energy Rev., vol. 15, no. 8, pp. 3757-3767, 2011.

[12] S. Ezzeldin and S. J. Rees, "The potential for office buildings with mixed-mode ventilation and low energy cooling systems in arid climates," Energy Build., vol. 65, pp. 368-381, Oct. 2013.

[13] K. Miura, Y. Takemasa, M. Katoh, and M. Hiraoka, "Effect of hybrid ventilation system on indoor environment and annual cooling load in a high-rise building," ASHRAE Transactions, vol. 118, pp. 831-846, 2012.

[14] P. Heiselberg, Principles of Hybrid Ventilation, Aalborg: Aalborg University, 2002.

[15] T. Kleiven, "Natural ventilation in buildings," Ph.D. dissertation, Department of Architectural Design, History and Technology, Norwegian University of Science and Technology, 2003.

[16] R. Z. Homod, K. S. M. Sahari, and H. A. F. Almurib, "Energy saving by integrated control of natural ventilation and HVAC systems using model guide for comparison," Renewable Energy, vol. 71, pp. 639-650, 2014.

[17] M. Mysen, J. P. Rydock, and P. O. Tjelflaat, "Demand controlled ventilation for office cubicles - can it be profitable?" Energy and Buildings, vol. 35, pp. 657-662, 2003.

[18] M. Mysen et al., "Occupancy density and benefits of demand-controlled ventilation in Norwegian primary schools," Energy and Buildings, vol. 37, pp. 1234-1240, 2005.

[19] D. Jreijiry, A. Husaunndee, and C. Inard, "Numerical study of a hybrid ventilation 20-system for single family houses," Solar Energy, vol. 81 , pp. 227-239, 2007.

[20] T. Lu, X. Lv, and M. Viljanen, "A novel and dynamic demand-controlled ventilation strategy for $\mathrm{CO}_{2}$ control and energy saving in buildings," Energy and Buildings, vol. 43, pp. 2499-2508, 2011.

[21] Z. Sun, S. Wang, and Z. Ma, "In-situ implementation and validation of a $\mathrm{CO}_{2}$-based adaptive demand-controlled ventilation strategy in a multi-zone office building," Building and Environment, vol. 46, pp. 124-133, 2011.

[22] J. D. Carrilho, B. Chenari, M. R. Gomes, G. Botte, M. Mateus, and M. G. da Silva, "Development of a live laboratory for research and technology demonstration in indoor environment assessment and control," in Proc. the 2nd Energy for Sustainability Multidisciplinary Conference - EfS 2015, Coimbra, Portugal.
[23] P. Michel and M. E. Mankibi, “Advanced control strategy,” 2000.

[24] P. O. Fanger, Thermal Comfort, Danish Technical Press, Copenhagen, 1970.

[25] ISO, "Ergonomics of the thermal environment - Analytical determination and interpretation of thermal comfort using calculation of the PMV and PPD indices and local thermal comfort criteria," International Standards Organization, Geneve, Switzerland, 2005.

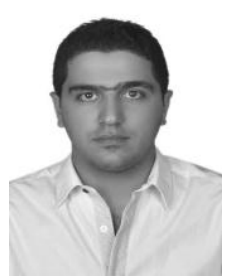

Behrang Chenari was born in 1986 in Iran. He graduated from Azad University of Tehran in mechanical engineering and then joined the Energy for Sustainability Master Program at University of Coimbra in September 2012. Currently, he is a Ph.D. candidate in sustainable energy systems, MIT Portugal Program, at University of Coimbra.

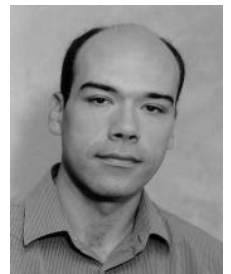

Joao Dias Carrilho was born in 1972, Lisbon, Portugal. He graduated in engineering acoustics and vibration at the University of Southampton, in 2001, and is currently a Ph.D. student in sustainable energy systems at the Energy for Sustainability Initiative of the University of Coimbra.

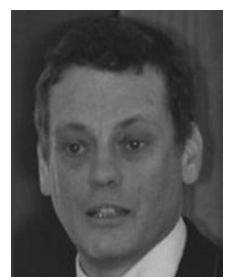

Manuel Gameiro da Silva was born in 1959, Coimbra, Portugal. He is an associate professor in the Department of Mechanical Engineering of the University of Coimbra, where they coordinates the Energy, Environment and Comfort Research Group of ADAI-LAETA. He is also a scholar of the MIT-Portugal Program and a member of the direction board of the Energy for Sustainability Initiative of the University of Coimbra. He is the vice-president of REHVA - Federation of European HVAC Associations. 